\title{
Growth kinetics and mechanism of glycine crystals
}

\author{
L. Li and N. Rodríguez-Hornedo ${ }^{1}$ \\ College of Pharmacy, The University of Michigan, Ann Arbor, Michigan 48109, USA
}

Received 14 January 1992

\begin{abstract}
Growth rate of the $\{001\}$ and $\{010\}$ faces of glycine crystals was measured as a function of supersaturation in a flow cell system. A growth mechanism was identified by comparing the results with theoretical models and by calculating the $\alpha$-factors. The BCF equation fits the growth data well. Thus, crystals growth of glycine can be explained by the screw dislocation mechanism.
\end{abstract}

\section{Introduction}

The control of crystal habit (shape) and growth rate has great importance in fields ranging from the chemical and pharmaceutical industries [1] to the crystallography of proteins and the laser technology using frequency-doubling crystals [2]. The shape of a crystals is determined by the relative growth rates of its faces. Faces with the slowest growth appear as large developed faces. The behavior of the growth rate of a crystal face is dependent on its underlying growth mechanism.

In a supersaturated solution, solute molecules are transported from the bulk of a solution to the crystal surface, and integrate into the surface at a kink site, or return to the solution. Depending on the rate limiting step, mechanisms can be classified into diffusion controlled or surface integration controlled. For surface integration controlled kinetics, the rate limiting steps are related to the structure of the solid-liquid interface. Several growth kinetic models have been proposed, and the dependence of growth rate $(G)$ on supersaturation $(\sigma=\ln (c / s))$ has been derived [3,4]. The interface structure can also be characterized in terms of the surface entropy factor, $\alpha$ [4]. Experimental results $[6,7]$ as well as Monte Carlo com-

1 To whom correspondence should be addressed. puter simulations [5] have shown that if $\alpha$ is less than about 3 , the interface is rough, and the growth is linearly dependent on the supersaturation. If $\alpha$ is greater than 4 , the interface is smooth, and the growth occurs at the steps generated by defects of surface nucleation.

We studied the growth kinetics of glycine crystals from aqueous solution. Glycine was chosen as a model compound because of: (1) simplicity of the molecular structure, (2) intermolecular hydrogen bonding in its crystal structure, which is similar to that found in peptides and protein crystals, and (3) the pro-chiral property of its crystal structure, which demonstrates an enantio-selectivity to chiral additives. A flow cell was designed to obtain the growth rates of each face of single crystals as a function of supersaturation under constant conditions (concentration and temperature). The growth mechanism was identified by fitting growth kinetic models to glycine growth data, and by calculating the $\alpha$-factors for each set of faces.

\section{Experimental procedure}

Materials: Glycine was obtained from Sigma.

Growth experiments: The growth rate was measured in the supersaturation range from 0.015 to 0.08 . A supersaturated solution was prepared by 


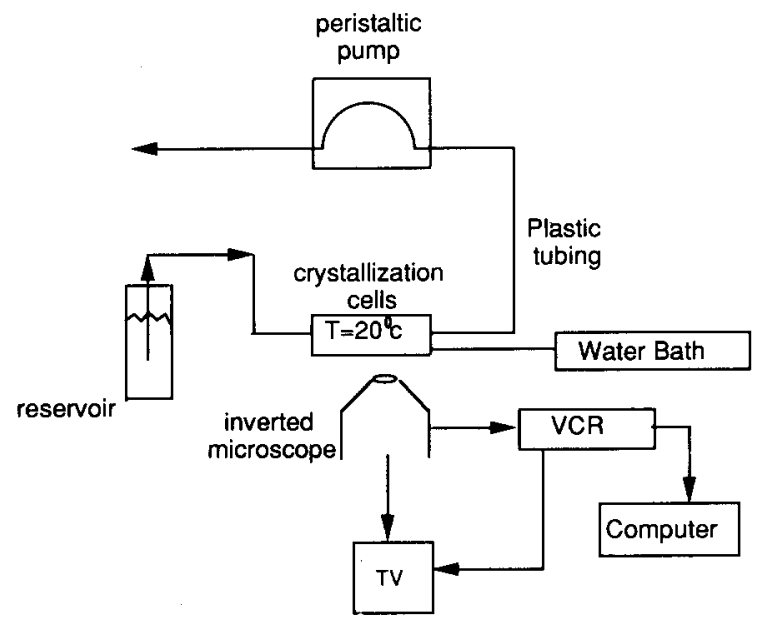

Fig. 1. Schematic diagram of the flow system used to measure growth rates of glycine crystals.

adding appropriate amounts of glycine and additives to water and heating the solution until all solids were dissolved. The hot solution $\left(\sim 80^{\circ} \mathrm{C}\right)$ was then filtered through a $0.45 \mu \mathrm{m}$ filter, and cooled to room temperature $\left(\sim 23^{\circ} \mathrm{C}\right)$. The concentration of glycine was then analyzed with a UV spectrophotometer (Perkin-Elmer).

The apparatus used for the flow cell experiments is shown in Fig. 1. A procedure for seed crystal preparation was developed that produced the desired size $(10-50 \mu \mathrm{m})$ and the number $(<10)$ of seeds in a cell. In this manner, local concentration gradients in the cells were avoided. Seed crystal preparation was as follows: A $40 \%$ ethanol-water solution saturated with glycine was mixed with the saturated glycine water solution (at $23^{\circ} \mathrm{C}$ ) in the ratio of $4: 1$. This solution was then placed into the cells. After glycine crystals nucleated, and the seeds grew to the desired size, the cells were flushed with the growth solution for approximately $15 \mathrm{~min}$ before growth rate measurements were started.

Supersaturated solutions were circulated by a peristaltic pump (WIZ, Isco) through the crystallization cells. The cells were made of glass tubing $(2 \times 4 \times 30 \mathrm{~mm})$ and were mounted in a water jacketed holder on an inverted microscope (Nikon Diaphot). The temperature of the cells was controlled by a water bath (Neslab) at $20 \pm 0.2^{\circ} \mathrm{C}$.
The size of crystals was measured by using $40 \times$ and $10 \times$ long-working-distance objectives.

Crystallographic structure analysis: Glycine crystals occur in three polymorphs. Single crystal $\mathrm{X}$-ray analysis (Syntex P2v and Siemens R3v) was used to determine the polymorphic form and the axes of the glycine crystals. A two-circle reflecting goniometer was used to identify the Miller indices of crystal faces.

\section{Results}

\subsection{Growth rate measurement}

Glycine crystals grown from water were bipyramidal (fig. 2). From single crystal $\mathrm{X}$-ray analysis, the $\alpha$-form of glycine crystals was obtained $\left(\mathrm{P} 2_{1} / \mathrm{n} ; a=5.10 \AA, b=12.0 \AA, c=5.46 \AA ; \beta=\right.$ $\left.111.7^{\circ} ; Z=4\right)$. The growth rates of $\{011\}$ and $\{010\}$ faces were measured for the crystals laying in a similar orientation to the one shown in fig. 2 in the crystallization cells. The size of the crystals was measured from the outline of crystals (fig. 3).

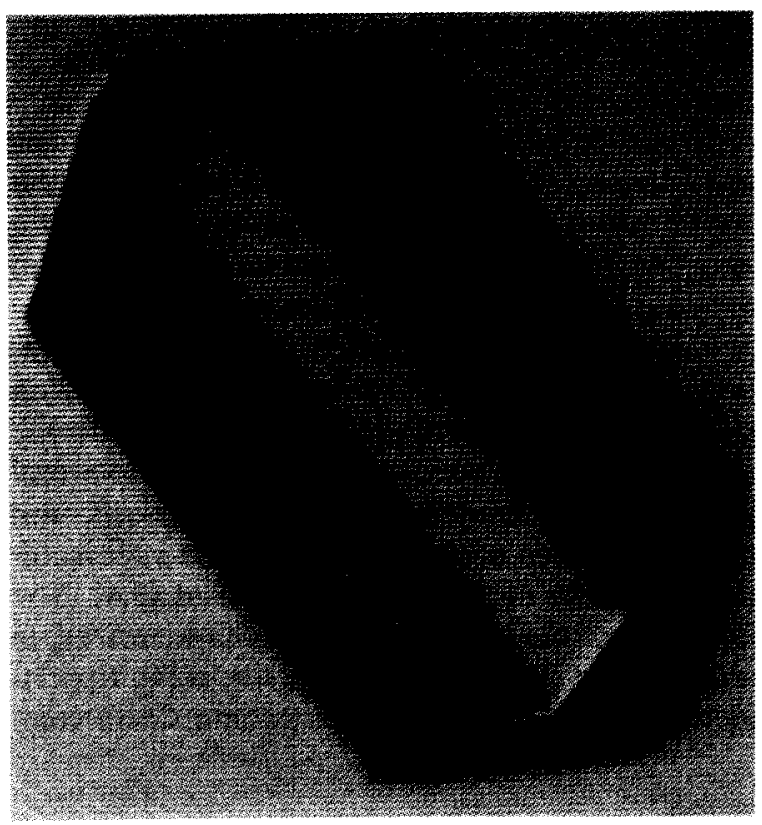

Fig. 2. Glycine crystal. 

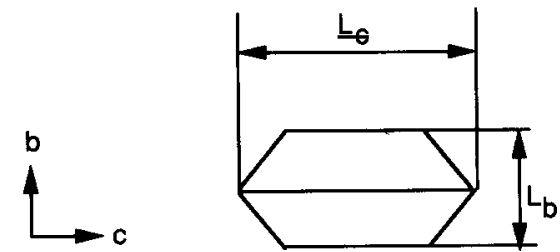

Fig. 3. Measurements of glycine crystals used to determine the growth rates of $\{011\}$ and $\{010\}$ faces.

The growth rate was calculated by

$$
\begin{aligned}
& G_{\{011\}}=\frac{1}{2} \sin 67.5^{\circ} \mathrm{d} L_{c} / \mathrm{d} t, \\
& G_{\{010\}}=\frac{1}{2} \sin 68.5^{\circ} \mathrm{d} L_{b} / \mathrm{d} t .
\end{aligned}
$$

The growth rate was measured as a function of supersaturation (fig. 4). The growth rate for each supersaturation in fig. 4 represents an average of several crystals ( $3-5$ crystals). For each crystal, 3 to 8 measurements of growth rates were done. It was observed that the larger seeds $(>100 \mu \mathrm{m})$ grew faster than the smaller ones. Thus, the growth rate data presented were obtained from a size range of 30 to $80 \mu \mathrm{m}$. A typical standard deviation of growth rate in a cell is $30 \%$, which may be partially due to the dispersion of growth rate.

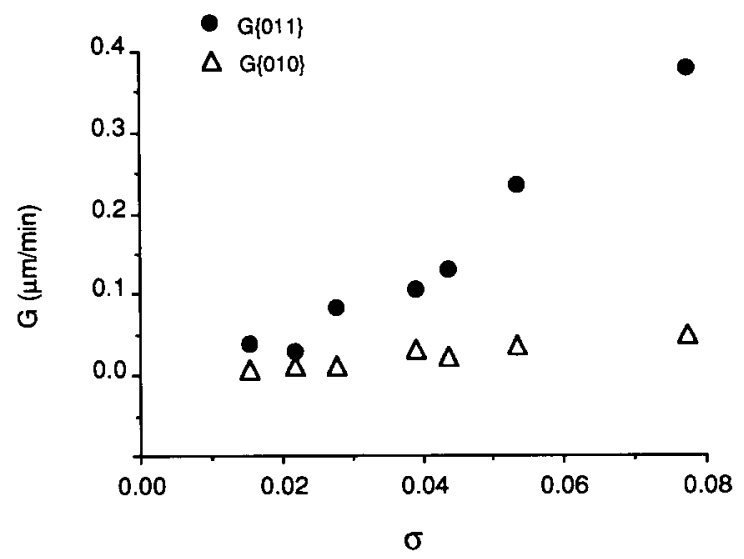

Fig. 4. Dependence of glycine growth rate $G$ on supersaturation $\sigma$.

\subsection{Kinetic models}

The flow rate used, $10 \mathrm{ml} / \mathrm{min}$, was within the range in which the growth rate was independent of the flow rate, and is not limited by diffusion in the bulk of the solution. Thus, the rate determining process of growth was the surface integration. Growth models have been developed, according to the number and the source of kink sites on the crystal surfaces, these include rough surface, screw dislocation, and surface nucleation mechanisms.

Rough surface mechanism: An interface is rough at the molecular level. Thus, there are many kink sites on the surface. In this case, a continuous growth equation has been proposed [8]:

$G=K_{1} \sigma$.

Screw dislocation mechanism (BCF model): The growth rate is limited by the integration of a growth unit into the crystal at a step generated by lattice defects on the surface. The dependence of growth rate on supersaturation has been described as [4]:

$$
G=K_{2} \sigma^{2} \tanh \left(K_{3} / \sigma\right),
$$

when $K_{3} \gg \sigma, \quad G=K_{2} \sigma^{2}$,

when $K_{3} \ll \sigma, \quad G=K_{4} \sigma$,

where $K_{4}=K_{2} K_{3}$.

Surface nucleation mechanism (birth and spread model): The rate limiting process of growth is the creation of a cluster with a size larger than the critical size. After a nucleus is formed, it grows at a finite rate by incorporation of growth units at steps. For this birth and spread model, the kinetic equation is written as [3]:

$G=K_{5} \sigma^{5 / 6} \exp \left(-\frac{\pi \gamma^{2}}{3\left(k_{\mathrm{B}} T\right)^{2} \sigma}\right)$,

where $k_{\mathrm{B}}$ is Boltzmann's constant, and $\gamma$ is the interfacial tension.

A semi-empirical growth rate equation,

$G=K \sigma^{a}$, 


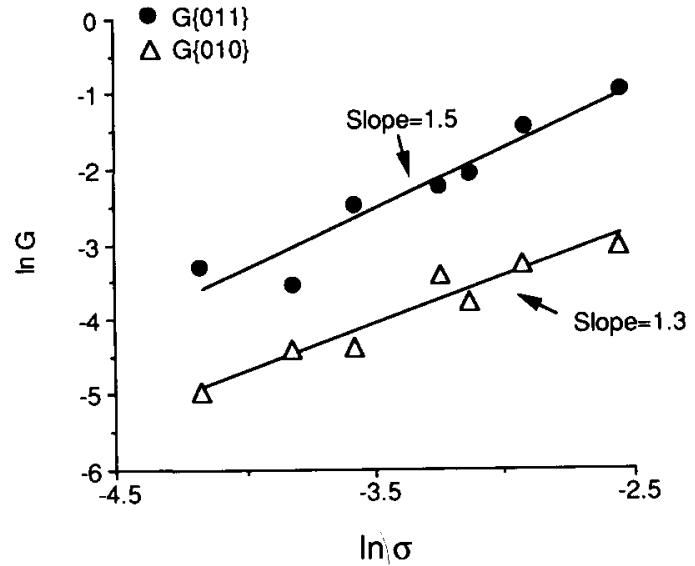

Fig. 5. Plot of growth rate of glycine crystals according to eq. (8).

frequently used in chemical engineering, was also tested. According to eqs. (4)-(6), $a=1$ for both the $\mathrm{BCF}$ model at high supersaturation and the rough surface mechanism, and $a=2$, for the BCF model at low supersaturation. If $a>2.5$, growth occurs by the surface nucleation mechanism. The values of $a$ were obtained by least square fit of the equation, $\ln G=a \ln \sigma+b$, to the data (fig. 5). It was found that $a=1.5 \pm 0.2$ for the set of $\{011\}$ faces, and $a=1.3 \pm 0.2$ for the set of $\{010\}$

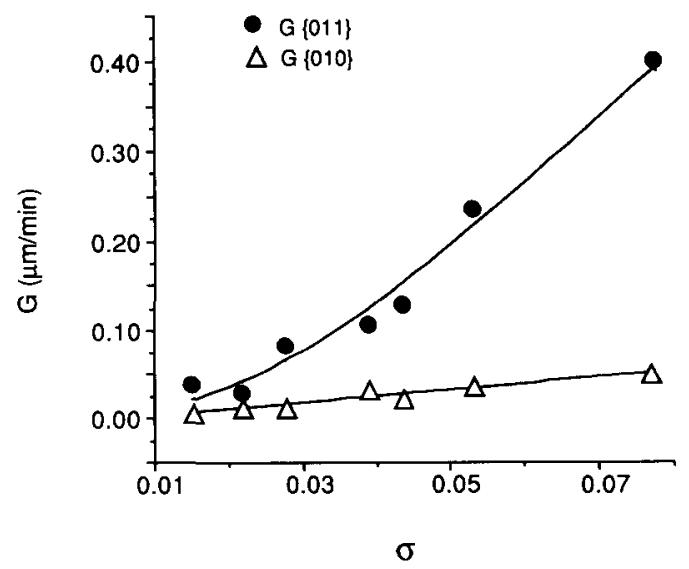

Fig. 6. Fit of the screw dislocation model (the solid lines) to the growth rate of glycine crystals obtained from non-linear regression.
Table 1

Parameter values from non-linear fit to eq. (4)

\begin{tabular}{lll}
\hline Faces & $K_{2}$ & $K_{3}$ \\
\hline$\{011\}$ & $84 \pm 8$ & $0.08 \pm 0.02$ \\
$\{010\}$ & $28 \pm 13$ & $0.03 \pm 0.02$ \\
\hline
\end{tabular}

faces. These results suggest that the growth rate data may be represented by the BCF model. The results of a non-linear fit of eq. (4) is shown in fig. 6 . The equations for glycine crystal growth rate $(\mu \mathrm{m} / \mathrm{min})$ are

$$
\begin{aligned}
& G_{\{011\}}=84 \sigma^{2} \tanh (0.08 / \sigma), \\
& G_{\{010\}}=28 \sigma^{2} \tanh (0.03 / \sigma) .
\end{aligned}
$$

The standard errors of the fitted parameters are listed in table 1.

To verify the mechanism, the growth data were further tested on the birth and spread model. The model was evaluated by plotting the data according to the linear form of eq. (7), $\ln \left(G / \sigma^{5 / 6}\right)$ versus $\sigma^{-1}$. As shown in fig. 7 , the data are not explained by this model. When surface nucleation occurred at higher supersaturation, the value of $\gamma$ obtained was an order of magnitude smaller than the value reported by Black and Davey [9].

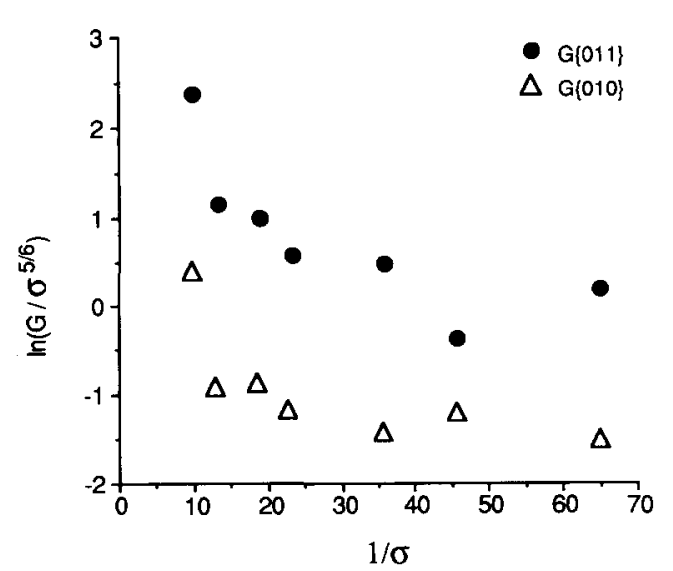

Fig. 7. Growth rate of glycine crystals plotted according to the surface nucleation model (eq. (7)). 


\subsection{The surface entropy factor}

In terms of measurable quantities, the $\alpha$-factor can be expressed as [10]:

$\alpha=\zeta \Delta H_{\mathrm{s}} / R T$,

where $\Delta H_{\mathrm{s}}$ is the heat of solution, and $\zeta$ is the anisotropy factor, and can be expressed as

$\zeta=E_{\text {slice }} / E_{\mathrm{cr}}$.

$E_{\text {slice }}$ is the horizontal bond energy between adjacent solid blocks, and $E_{\text {cr }}$ is the total crystallization energy.

Using eq. (11), the $\alpha$-factor was calculated to be 3.9 for the $\{011\}$ faces and 5.5 for the $\{010\}$ faces, based on the heat of solution [11] and attachment and slice energies [12]. The observations of faceted faces and the high value of the $\alpha$-factor suggest that the growth occurs on a smooth surface by a defect mediated mechanism. This is in agreement with the results from the growth kinetics.

\section{Discussion}

According to the results from fitting various models to the glycine crystal growth data and the calculation of the $\alpha$-factor, the growth of glycine crystals is explained by the screw dislocation mechanism. The parameter, $K_{3}$, in eq. (4) was found to be 0.08 for the $\{011\}$ faces and 0.03 for the $\{010\}$ faces. These values of $K_{3}$ are in the same range as the experimental values of the supersaturation (0.015-0.08). In this case, eq. (4) cannot be simplified to either the quadratic function in eq. (5) or the linear function in eq. (6). This is consistent with the fitted $a$-values in eq. (8) (1.5 for the $\{011\}$ faces and 1.3 for the $\{010\}$ faces).

Only thermodynamic variables are taken into account in the $\alpha$-factor; however, the kinetic aspects and the adsorption of solvent molecules on the crystal surface should also be considered. The crystal structure of glycine (fig. 8) [16] shows that the $\{011\}$ faces are very polar. On the $\{011\}$ faces, both oxygen atoms of the carboxyl group are

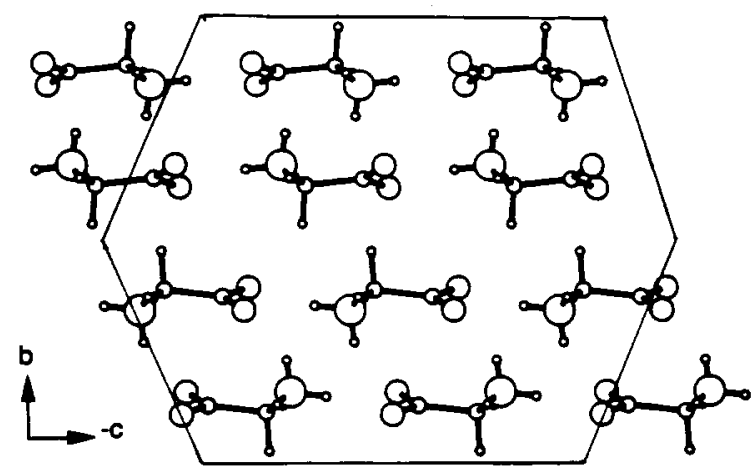

Fig. 8. Packing arrangement of $\alpha$-glycine viewed along the $a$ axis.

normal to the faces. This provides good binding sites for polar water molecules [12]. Thus, the growth of $\{011\}$ faces, along the $c$ direction, is expected to be slower than the predicted rate based on the $\alpha$-factor. Although the calculated $\alpha$-factor value, 3.9 , for the $\{011\}$ face is at the border of the BCF model and other mechanisms, the slower growth rate of $\{011\}$ faces due to solvent adsorption supports our proposition of the screw dislocation mechanism for the glycine crystal growth.

Our observation that the larger crystals often grew faster than the smaller ones under the same conditions may be related to the growth dispersion. Growth dispersion of single crystals under constant conditions has been reported in several studies [13-15]. This phenomenon may indicate a surface integration mechanism, particularly the BCF model, where the variation in growth rates is due to the difference in the surface density of defects on the seed crystals.

\section{Acknowledgments}

We thank Jeff W. Kampf in the Department of Chemistry, The University of Michigan, for single crystal X-ray analysis. This work was supported by the National Science Foundation (DMB8818627). 


\section{References}

[1] A.S. Myerson and K. Toyokura, Eds., Crystallization as a Separations Process, Am. Chem. Soc. Symp. Ser., Vol. 438 (1990).

[2] A.A. Chernov, Contemp. Phys. 30 (1989) 251.

[3] M. Ohara and R.C. Reid, Modelling Crystal Growth Rates from Solution (Prentice-Hall, Englewood Cliffs, NJ, 1973).

[4] P. Bennema and G.H. Gilmer, in: Crystal Growth: An Introduction, Ed. P. Hartman (North-Holland, Amsterdam, 1973) pp. 263-327.

[5] G.H. Gilmer and P. Bennema, J. Crystal Growth 13/14 (1972) 148.

[6] P. Bennema and J.P. van der Eerden, J. Crystal Growth 42 (1977) 201.
[7] J.R. Bourne and R.J. Davey, J. Crystal Growth 36 (1976) 287.

[8] J.D. Weeks and G.H. Gilmer, Advan. Chem. Phys. 40 (1979) 157.

[9] S.N. Black and R.J. Davey, J. Crystal Growth 90 (1988) 136.

[10] P. Bennema and J.P. van der Eerden, in: Morphology of Crystals, Part A, Ed. I. Sunagawa (Terra, Tokyo and Reidel, Dordrecht, 1987) p. 32.

[11] B.P. Kelley and T.H. Liley, J. Chem. Thermodynamics 10 (1978) 703.

[12] Z. Berkovitch-Yellin, J. Am. Chem. Soc. 107 (1985) 8239.

[13] J. Garside and R.I. Ristić, J. Crystal Growth 61 (1983) 215.

[14] J. Garside, AICHE Symp. Ser. 80 (1982) 23.

[15] A.V. Valčić, J. Crystal Growth 30 (1975) 129.

[16] R.E. Marsh, Acta Cryst. B 11 (1958) 654 